\title{
Fully automated macromolecule suppressed single voxel glutamate spectroscopy (FAMOUS SVGS)
}

\author{
Ravi Prakash Reddy Nanga*, Hari Hariharan and Ravinder Reddy
}

\begin{abstract}
Purpose: The aim of the study was to develop and validate a new localized ${ }^{1} \mathrm{H}$ MRS pulse sequence and automated post-processing software for the quantification of brain Glutamate (Glu) in clinical conditions at 7.0T in order to get reliable and reproducible results for acute intervention studies.

Methods: Here we describe a new localized proton MRS method "Fully Automated MacrOmolecUle Suppressed Single Voxel Glutamate Spectroscopy (FAMOUS SVGS)" for measuring Glu. FAMOUS SVGS method consists of a new pulse sequence with optimized switchable water, metabolites and outer volume suppression modules, as well as a frequency selective inversion pulse and automated post-processing of the five spectra obtained. FAMOUS SVGS method was first validated with glutamate phantoms and then validated with test-retest repeatability studies in the occipital cortex of five normal volunteers at 7.0T.

Results: Glutamate concentrations estimated from phantoms with FAMOUS SVGS method correlated well with actual concentrations. Test-retest repeatability studies in human brain in vivo yielded less than $0.3 \mathrm{mM}$ intra-subject variations in Glu concentrations.

Conclusions: FAMOUS SVGS method enables Glu quantification in vivo at 7.0T with test-retest variability of less than $0.3 \mathrm{mM}$. We expect that we can reliably measure $\geq 0.5 \mathrm{mM}$ change in glutamate due to any acute intervention.
\end{abstract}

Keywords: Single voxel ${ }^{1} \mathrm{H}$ MRS, Glutamate quantification

\section{Background}

Glutamate (Glu) is a major excitatory neurotransmitter that is present in abundance in the synaptic vesicles of the human brain [1]. It plays an important role in the day-to-day functions such as cognitive, learning and memory, a key metabolite in cellular metabolism, and helps in removal of ammonia from the body. Its quantification has gained significant importance due to its implications in many of the neuropsychological as well as neurodegenerative disorders. In these brain disorders, as the changes in the Glu levels are expected to be relatively small, it is important to have a non-invasive method that

\footnotetext{
*Correspondence: nravi@mail.med.upenn.edu

Department of Radiology, Center for Magnetic Resonance and Optical Imaging, University of Pennsylvania, B1 Stellar-Chance Laboratories, 422 Curie Boulevard, Philadelphia, PA 19104-6100, USA
}

measures these small changes in Glu concentration with high degree of accuracy and repeatability.

Single voxel proton magnetic resonance spectroscopy $\left({ }^{1} \mathrm{H}\right.$ MRS) which is a safe, non-invasive method for the detection of human brain metabolites in vivo, has been widely applied for the study of Glu levels in many of the neurological disorders as well as in cases of insomnia and addiction disorders [2-14]. With the availability of 7.0T ultra-high field magnets, and the phased-array head coils, there is an improved resolution in the ${ }^{1} \mathrm{H}$ MRS and appreciable gain in signal-to-noise ratio (SNR) over the lower field strength magnets [15]. In a previous acute gabapentin drug study done at 7.0T, the effect of drug produced detectable change in GABA but not for glutamate [16]. Based on literature reports and from our own experience, we feel the following issues might contribute to the glutamate quantification errors: (i) Eddy currents, 
(ii) Phased-array combination from the multi-channel receive head coils, (iii) Chemical shift artifacts, (iv) Phase correction, (v) Baseline correction, and (vi) Macromolecular contaminations. There are few test-retest studies done at 7.0T, where mean inter-subject variations in glutamate estimation are reported to be around $5 \%[17,18]$, but intra-subject test-retest variation for each individual in glutamate estimation is not reported. Our interest is in detecting the absolute quantification of glutamate for pre- and post acute interventions of an individual with an aim to detect $\geq 0.5 \mathrm{mM}$ change in glutamate concentrations in clinical conditions such as temporal lobe epilepsy, cortical dysplasia, Alzheimer's, Psychiatric disorders, etc.

This has motivated us to develop a new strategy to design a new pulse sequence as well as develop an automated processing pipeline with no user intervention to mitigate the above-mentioned issues with the single voxel ${ }^{1} \mathrm{H}$ MR spectra, for improved repeatability of the Glu measurement.

In this study, we demonstrate a new method for quantification of the Glu at $2.35 \mathrm{ppm}$ from the Fully Automated MacrOmolecUle Suppressed Single Voxel Glutamate Spectroscopy (FAMOUS SVGS). FAMOUS SVGS ${ }^{1} \mathrm{H}$ MRS with an automated post-processing provides a simple spectrum with a flat baseline. FAMOUS SVGS method was validated in vitro using glutamate monosodium phantoms. We investigated in vivo efficacy of this method by acquiring the data from the voxel in occipital cortex from multiple subjects, at two-time points.

\section{Methods}

\section{FAMOUS SVGS sequence}

FAMOUS SVGS pulse sequence is shown in Fig. 1. The sequence consists of a water reference acquisition, two control acquisitions with selective inversion set at $7.05 \mathrm{ppm}$ ["water suppressed" (Control1) and "water and metabolite suppressed" (Control2)], and two selective inversion acquisitions set at $2.35 \mathrm{ppm}$ ["water suppressed" (Selinv1) and "water and metabolite suppressed" (Selinv2)]. The number of averages for the water reference acquisition and water-suppressed acquisitions can be independently set. The basic sequence has a magnetization preparation and single voxel acquisition blocks. The preparation block consists of double inversion for metabolite suppression, water suppression, outer volume suppression (OVS) and frequency selective inversion (FSI). The acquisition block consists of the standard PRESS sequence. The double inversion segment is designed with broad band adiabatic inversion pulses that do not excite water (pulse width $=18 \mathrm{~ms}$, bandwidth $=3000 \mathrm{~Hz}$ (10 ppm centered at $-1 \mathrm{ppm}$ ). The inversion delays are optimized to provide good inversion for metabolite $T_{1}$ values between 1 and $2 \mathrm{~s}$. Water suppression is performed using seven water suppression pulses $(\alpha 1-\alpha 7)$; Gaussian shape with user-defined bandwidth and seven inter-pulse delays (d1-d7). Delay $\mathrm{d} 4$ is designed to be long enough $(>20 \mathrm{~ms})$ to accommodate up to 4 OVS pulses $(3.5 \mathrm{~ms}$ radio frequency $(\mathrm{RF})$ pulse (bandwidth $=5600 \mathrm{~Hz}$ ) and $1.3 \mathrm{~ms}$ gradient spoiler). Delay $\mathrm{d} 7$ is designed to be long enough ( $>45 \mathrm{~ms}$ ) to accommodate both the OVS and FSI pulses. The FSI pulse is a Gaussian pulse with its width calculated to provide $50 \%$ inversion over a user defined inversion bandwidth (typically $60 \mathrm{~Hz}$ to cover the distance between the center peak of the triplet to the either side $(7 \mathrm{~Hz}+7 \mathrm{~Hz})$ and an additional $45 \mathrm{~Hz}$ for shim inhomogeneities). The water reference acquisition is performed with all spoiler gradients and OVS RF pulses without any water suppression, double inversion or FSI pulses. This is essential since we use the water reference spectrum to correct eddy current induced dynamic phase shifts for all the five spectra. "Water suppressed" spectra are acquired with water suppression, OVS and FSI pulses. "Water and metabolite suppressed" spectra are obtained with double inversion, water suppression, OVS and FSI pulses. The FSI chemical shift (invppm) is user selectable (for Glu, invppm is $2.35 \mathrm{ppm}$ ) and the sequence automatically calculates the offset frequencies for the control and Selinv acquisitions to be symmetrically placed around water resonance (at $4.7+2.35 \mathrm{ppm}$ ) and at $2.35 \mathrm{ppm}$ respectively. Inversion at $(4.7+2.35 \mathrm{ppm})$ is done to remove any residual baseline water difference. For the SVS PRESS block, custom designed Shinnar-Le Roux (SLR) RF pulses, were used for uniform excitation and refocusing profile [19]. Relevant SLR pulse design parameters are: (a) excitation pulse: pulse width $=2.7 \mathrm{~ms}$, time bandwidth product $=9$, pass band ripple $=1 \%$, stop band ripple $=0.2 \%$, flip angle $=90^{\circ}$, and (b) refocusing pulse: pulse width $=6 \mathrm{~ms}$, time bandwidth product $=12$, pass band ripple $=1 \%$, stop band ripple $=0.1 \%$, flip angle $=150^{\circ}$. In the PRESS part of the sequence, for the water acquisition the excitation and refocusing pulses were set on resonance with water peak at $4.7 \mathrm{ppm}$ while for all "water-suppressed" and "water and metabolite" spectra, the excitation and refocusing pulses were set at the glutamate chemical shift of $2.35 \mathrm{ppm}$ to avoid chemical shift artifact for glutamate. This chemical shift based offset was added to the gradient strength and positionbased frequency offset for all PRESS pulses. This ensures that the signal for water reference, Control1, Control2, Selinv1 and Selinv2 spectra all originate from the same location. The scan protocol uses a smaller number of averages for the water reference acquisition block and a larger number of averages for the water-suppressed and water-suppressed metabolite nulled acquisition blocks to optimize scan time. 


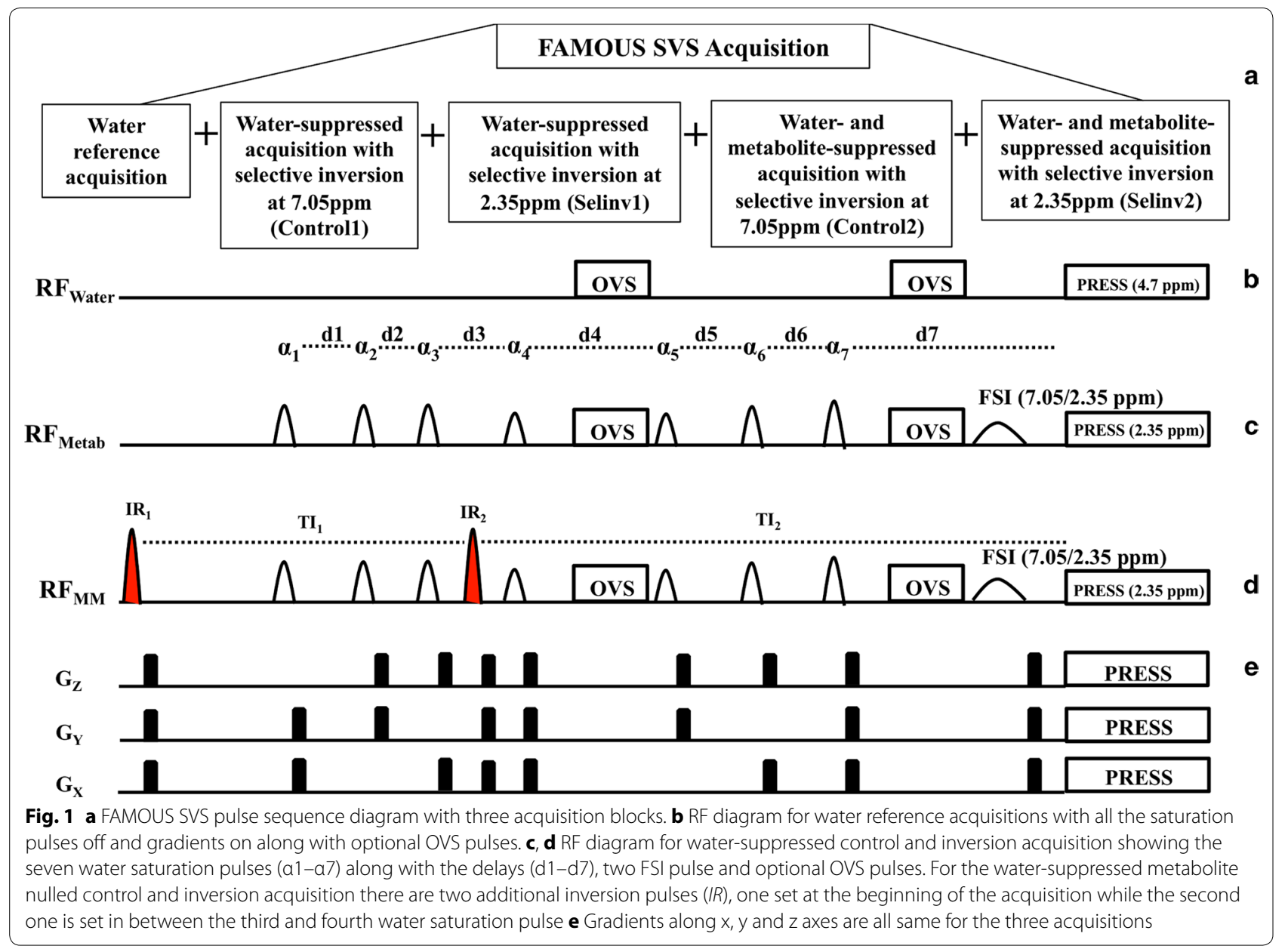

\section{MR scan protocol}

All the single voxel ${ }^{1} \mathrm{H}$ MRS experiments were performed on a 7.0T whole body MRI scanner (Siemens Medical Systems, Erlangen, Germany) with a vendor supplied volume coil transmit/32-Channel receive proton head phased array coil. The acquisition protocol consisted of a gradient echo localizer, a $\mathrm{T}_{1}$-weighted three-dimensional (3D) magnetization prepared rapid gradient echo (MPRAGE) of whole brain. Localized automated first order and second order shimming of the $\mathrm{B}_{0}$ field was performed on the voxel of interest in order to obtain a water line width of $<15 \mathrm{~Hz}(0.05 \mathrm{ppm})$ or less using FASTMAP shim method $[20,21]$ provided by Siemens as work in progress (WIP) package. Optimal reference voltage for the region of interest was calculated by acquiring two water spectra with reference voltages of 100 and $200 \mathrm{~V}$ using stimulated echo acquisition mode (STEAM) sequence. Assuming the peak amplitudes of water signal to be $\mathrm{S} 1$ and $\mathrm{S} 2$ for the above two reference voltages, the optimal reference voltage "B1" for the region of interest was calculated using the following equation:

$$
B 1=\frac{90 * 100}{\frac{180}{\pi} * \operatorname{acos}\left(\sqrt[3]{\frac{S 2}{8 * S 1}}\right)}
$$

Single voxel ${ }^{1} \mathrm{H}$ MRS was obtained with FAMOUS SVGS sequence.

\section{Phantom studies}

We verified the accuracy of the FAMOUS SVGS method using the glutamate monosodium phantoms. A stock solution of $100 \mathrm{mM}$ Glutamate monosodium phantom was prepared using PBS buffer. From this stock solution, four phantoms were prepared at physiological $\mathrm{pH}$ to give final concentrations of $6,9,12,15 \mathrm{mM}$. Even though with phantoms we could optimize shims to get line widths $<5 \mathrm{~Hz}$, to mimic the in vivo conditions, shim currents were mis-set to obtain a line width comparable to that of in vivo $(15 \mathrm{~Hz})$. Single voxel ${ }^{1} \mathrm{H}$ MR spectra were obtained on the phantom with FAMOUS SVGS at room temperature using following parameters: voxel-size $=20 \times 30 \times 20 \mathrm{~mm}^{3}$, repetition time 
$(\mathrm{TR})=3000 \mathrm{~ms}$ and time to echo $(\mathrm{TE})=21 \mathrm{~ms}$, number of points $=4096$, sweep width $=6000 \mathrm{~Hz}$, dummy scans $=4$, averages $=8$ (water) $/ 32$ (Control 1) $/ 32$ (Selinv1). Total acquisition time to obtain the spectra was $4 \mathrm{~min}: 12 \mathrm{~s}$ per phantom.

\section{Human studies}

\section{Ethics, consent and permission}

All the human studies were conducted under an approved University of Pennsylvania Institutional Review Board protocol with written informed consent obtained from each volunteer after explaining the study protocol.

Voxel was positioned in the occipital cortex (OCC, $20 \times 30 \times 20 \mathrm{~mm}^{3}$ ) with saturation band covering the skull area and the spectra was obtained with the following parameters: repetition time $(\mathrm{TR})=3000 \mathrm{~ms}$ and time to echo $(\mathrm{TE})=21 \mathrm{~ms}$, number of points $=4096$, sweep width $=6000 \mathrm{~Hz}$, dummy scans $=4$, averages $=8$ (water), 32 (Control 1), 32 (Selinv1), 32 (Control 2), 32 (Selinv2).
Total acquisition time to obtain the spectra was $7 \mathrm{~min}: 48 \mathrm{~s}$. All the five volunteers (all males, aged 19-62 years) were scanned twice within day for repeatability studies.

\section{Voxel placement for studies involving multiple scans}

We used our in-house developed software, "Imscribe", described elsewhere [22] to maintain the consistency in voxel placement for multiple acquisitions of ${ }^{1} \mathrm{H}$ MRS from the same voxel.

Imscribe (accessible at: cmroi.med.upenn.edu/ imscribe), uses high resolution $\mathrm{T}_{1}$-weighted images and the voxel information from the spectroscopy file acquired from first scan as target template for subsequent scans and performs affine co-registration giving the information for the new voxel placement as illustrated in Fig. 2.

\section{MRS data processing}

For spectroscopy post-processing, we have an in-house developed automated post-processing package using

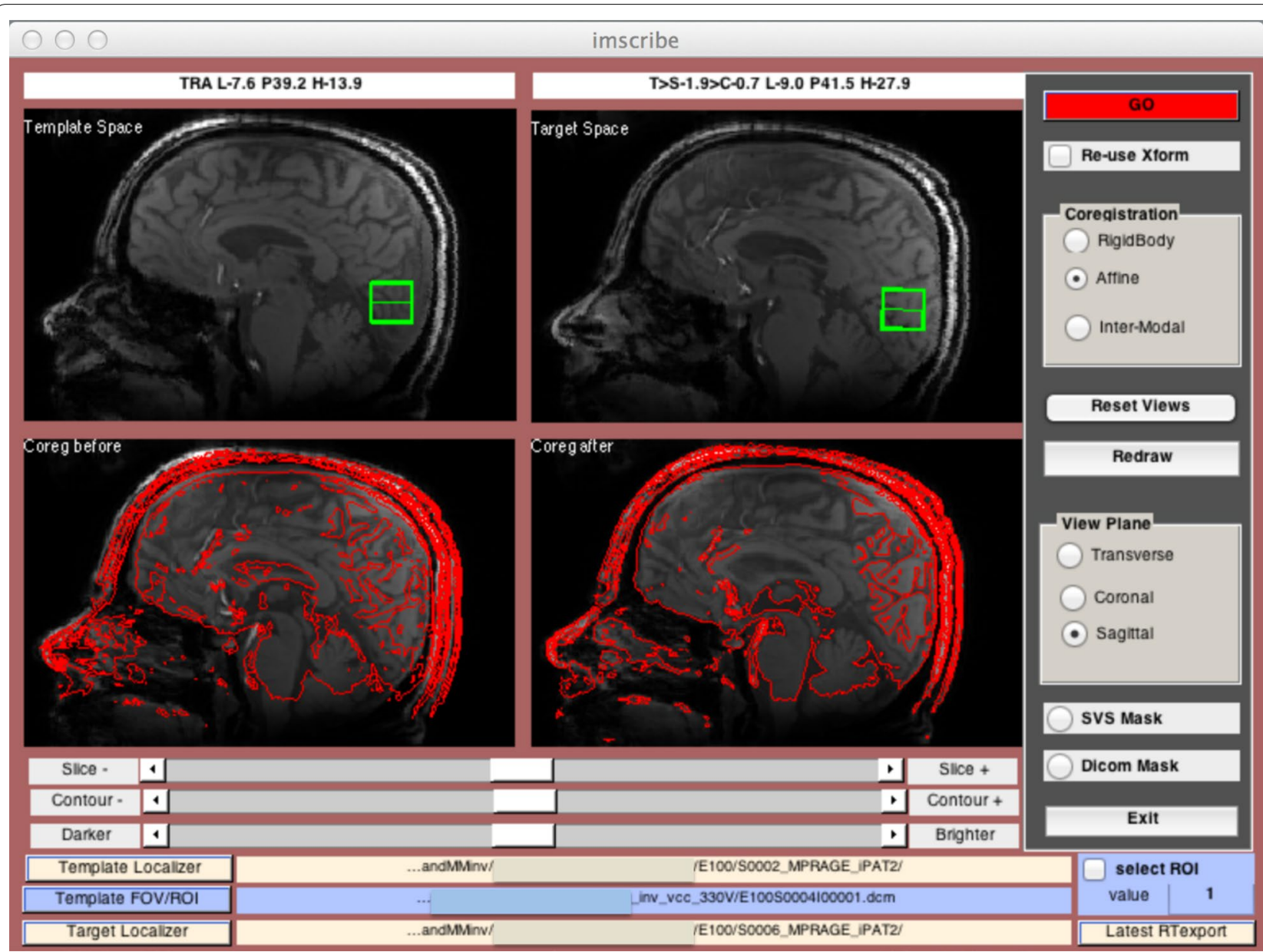

Fig. 2 Snap-shot of the "Imscribe" software showing the co-registration process of the template and target $T_{1}$-weighted images along with the new voxel information in the target space 
MATLAB (R2014a, MathWorks, Natick, MA). Postprocessing was done on the raw data from the system before any phased array channel combination and the flow chart is depicted in Fig. 3. For the phantom spectra, the FAMOUS Glu spectrum was obtained as (Control 1-Selinv1) and fitted as Lorentzian. Water content was assumed to be $100 \%$ for phantoms.

In-house developed spectral processing method for FAMOUS SVGS works in the frequency domain. In this method, an exponential apodization of $\sim 7 \mathrm{~Hz}$ was applied to the final time domain data after taking care of eddy current induced phase shifts, followed by automated Fourier transformation, phase correction and macromolecule removal. For fitting of water reference spectrum, we used non-linear least squares sense fitting (MATLAB "lsqcurvefit" routine). The integral of the Glu peak from 2.25 to $2.45 \mathrm{ppm}$ (based on the inversion pulse bandwidth of $60 \mathrm{~Hz}$ ) was normalized by water reference integral for absolute quantification of Glu. Water content was assumed to be $80 \%$ for human brains.

\section{Statistics}

Repeatability intra-class correlation coefficient (ICC) with $95 \%$ confidence intervals $(\mathrm{CI})$ values of single measures for the test-retest data was calculated with one-way random model using the SPSS software [version 18]. ICC value of $<0.0$ is interpreted as poor agreement, $0.0-0.2$ indicates slight agreement, $0.21-0.4$ indicates fair agreement, $0.41-0.6$ indicates moderate agreement, $0.61-0.8$ indicates substantial agreement, whereas ICC $>0.81$ indicates an almost perfect agreement [23]. ICC with p value less than 0.05 is considered as statistically significant.
Reading the five time domain (FID) raw data sets with all channel data separately.<smiles>C1CC2CC12</smiles>

Using the first point of the water FID, we get the relative amplitudes of the each channel and choose the signals from those channels which have $>5 \%$ of the channel with maximum signal and use those channels for further processing.

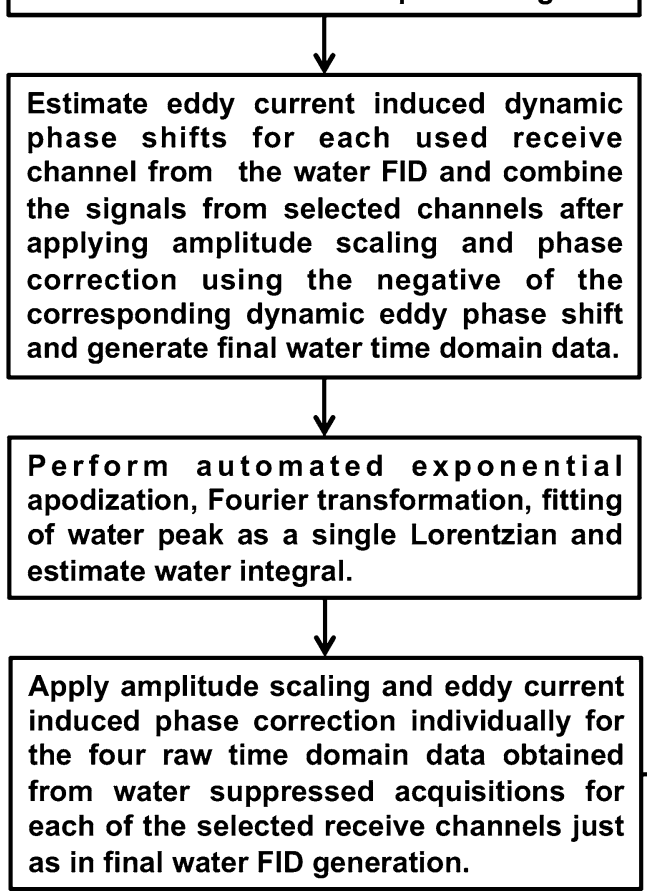

Glu concentartion is estimated from the integral between 2.25 to $2.45 \mathrm{ppm}$ of FAMOUS GIu spectrum for absolute quantification.

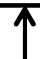

Final FAMOUS Glu spectrum is obtained as (Control1 - Selinv1) - (Control2 Selinv2).

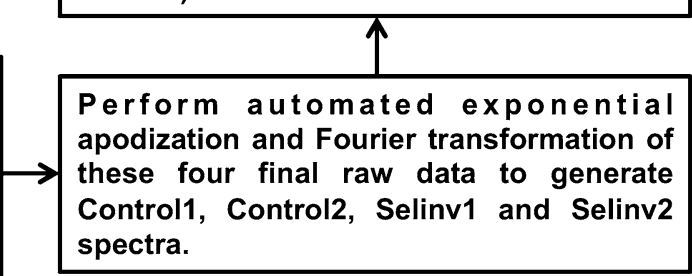

Fig. 3 Flow chart showing the automated post-processing steps involved in quantification of the raw data obtained from the application of FAMOUS SVGS 


\section{Results}

The concentration of glutamate from the voxel positioned on the glutamate monosodium phantom, as shown in Fig. 4, from the FAMOUS SVGS shows a good linear correlation between the actual and measured glutamate concentrations (slope $=1.03$, goodness of fit as measured by $R^{2}=0.997$ ).

Figure 5 shows the typical water spectrum obtained from the volunteer in OCC voxel and the typical Control1 and Control 2 spectra. One can clearly see a 140fold reduction in water signal in the later two spectra and metabolite suppression has very little effect on the quality of water suppression.

Figure 6 shows the OCC voxel position and the typical Control1, Control2, (Control1-Selinv1), (Control2Selinv2) and FAMOUS Glu spectra from a volunteer. In the inset is shown the FAMOUS Glu spectrum along with the inversion profile of the FSI pulse. FAMOUS Glu spectrum is integrated from 2.25 to $2.45 \mathrm{ppm}$, which is based on the inversion bandwidth of the FSI pulse as shown in inset of Fig. 6.
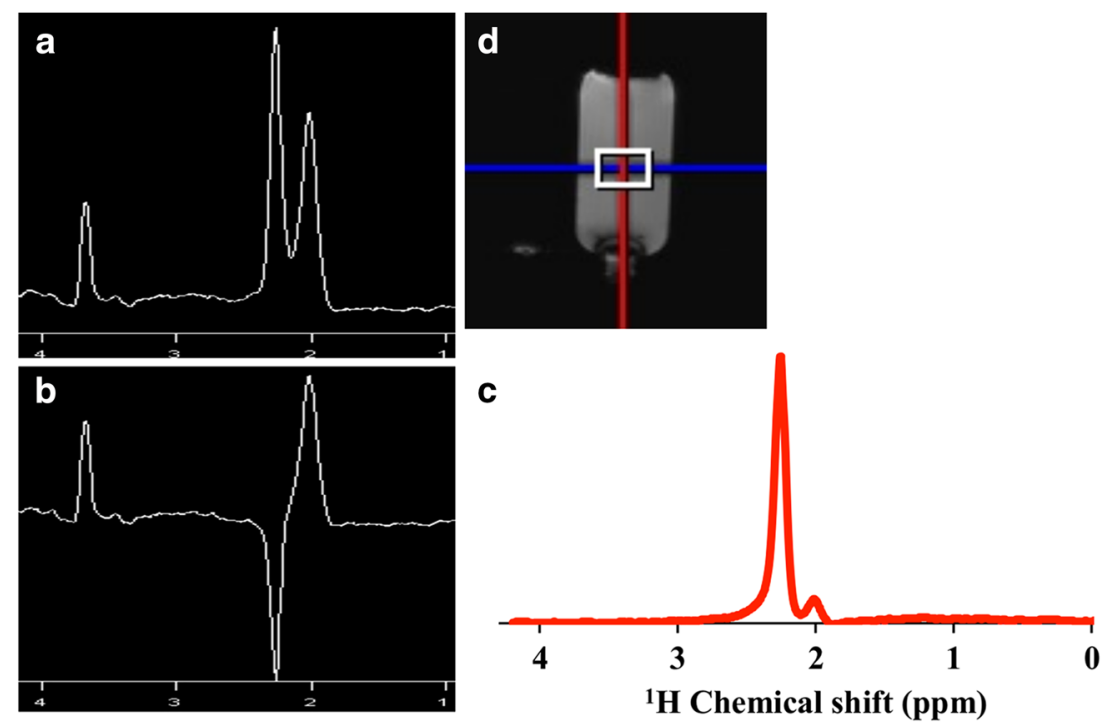

e

f

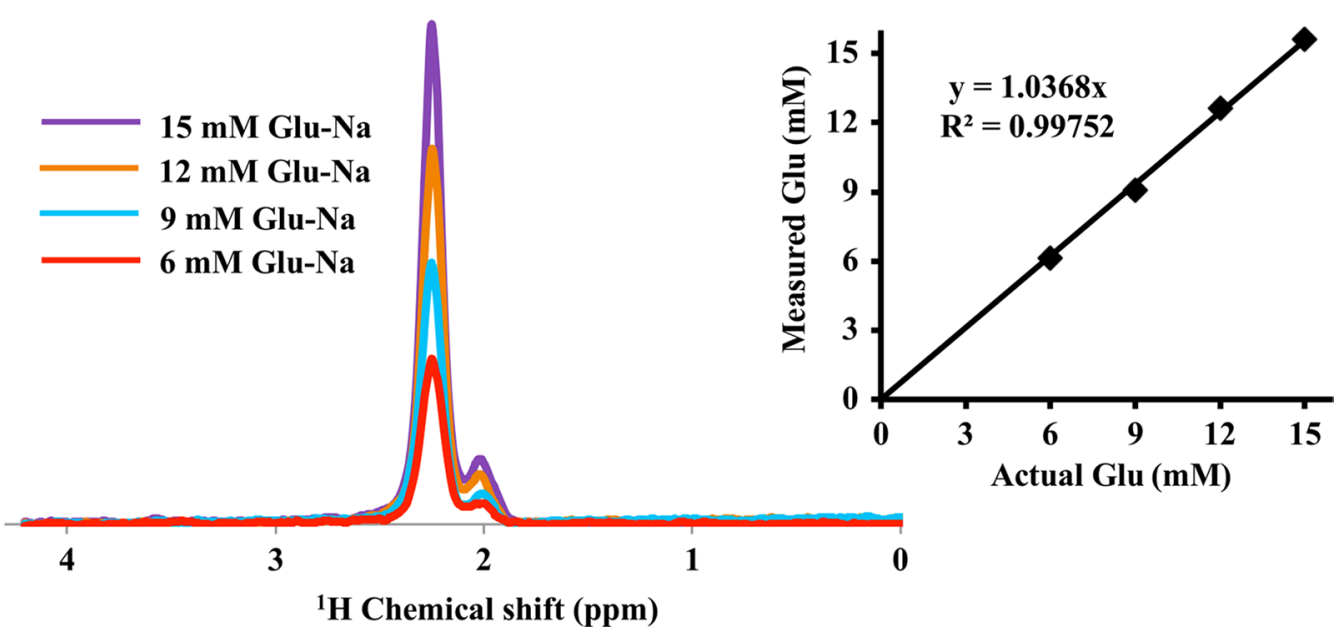

Fig. 4 Water-suppressed control spectrum (a) and water-suppressed inversion spectrum (b) along with the FAMOUS Glu spectrum (c) obtained on region of interest shown on glutamate monosodium phantom image (d) along with the FAMOUS Glu spectra obtained from different concentrations of glutamate monosodium phantom (e) and a plot of actual vs measured Glu concentration (f) from FAMOUS Glu spectra 


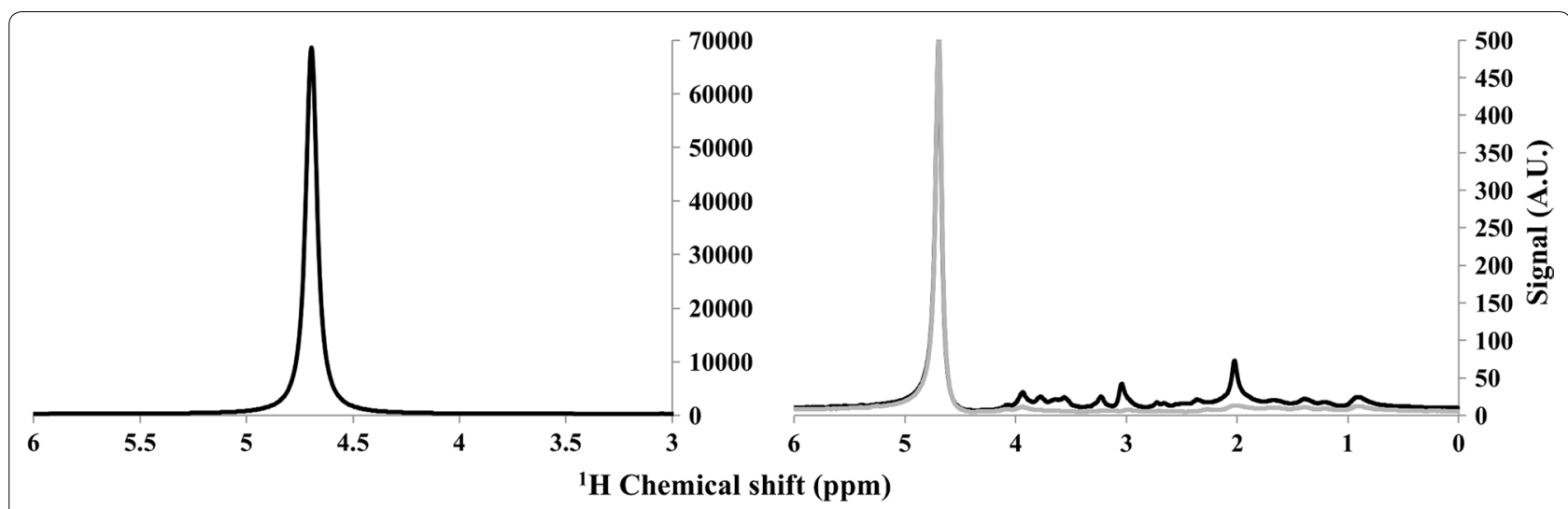

Fig. 5 Typical water reference spectrum from one of the volunteer on OCC voxel along with the Control1 (black) and Control2 (grey) spectra demonstrating a good water suppression in the later two spectra. This suppression of water peak was 140-fold

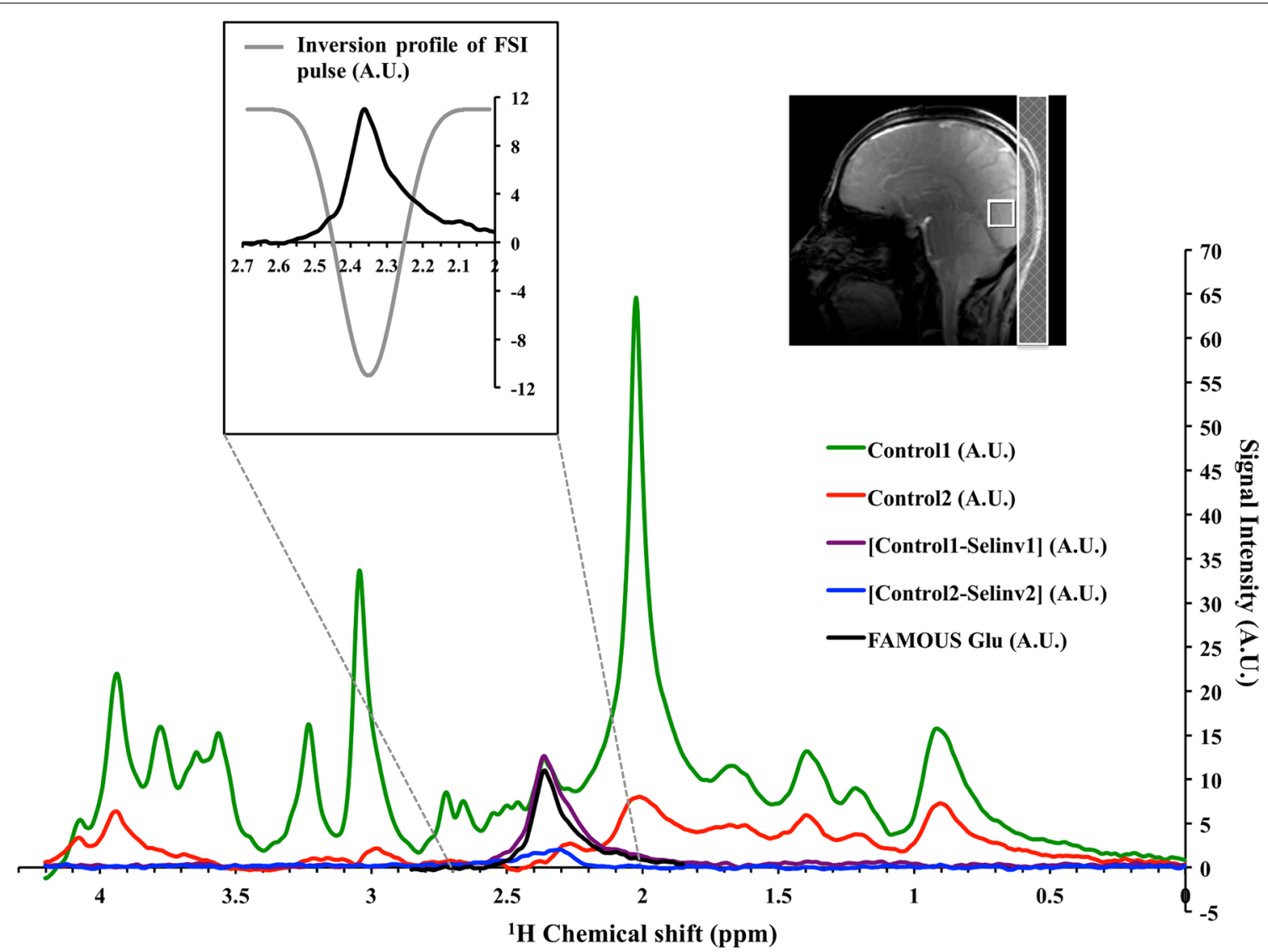

Fig. 6 Anatomical position of the voxel on OCC from a volunteer is shown in $\mathrm{T}_{1}$-weighted image along with Control1 (green), Control2 (red), [Control1-Selinv1] (brown), [Control2-Selinv2] (blue) and FAMOUS Glu (black) spectra. In the inset is shown FAMOUS Glu spectrum along with the inversion profile (grey) of the FSI pulse at $2.35 \mathrm{ppm}$

FAMOUS Glu spectra of test-retest of all the five subjects for each scan are shown in Fig. 7. The mean and standard deviation of Glu concentration from FAMOUS Glu spectra for the ten scans obtained from the five volunteers in the OCC voxel is $9.57 \pm 0.63 \mathrm{mM}$; $[\mathrm{CoV}=6.6 \%$ calculated as (standard deviation/mean) $\times 100]$.

Absolute changes in Glu variations for each volunteer on OCC voxel are summarized in Table 1. The maximal 

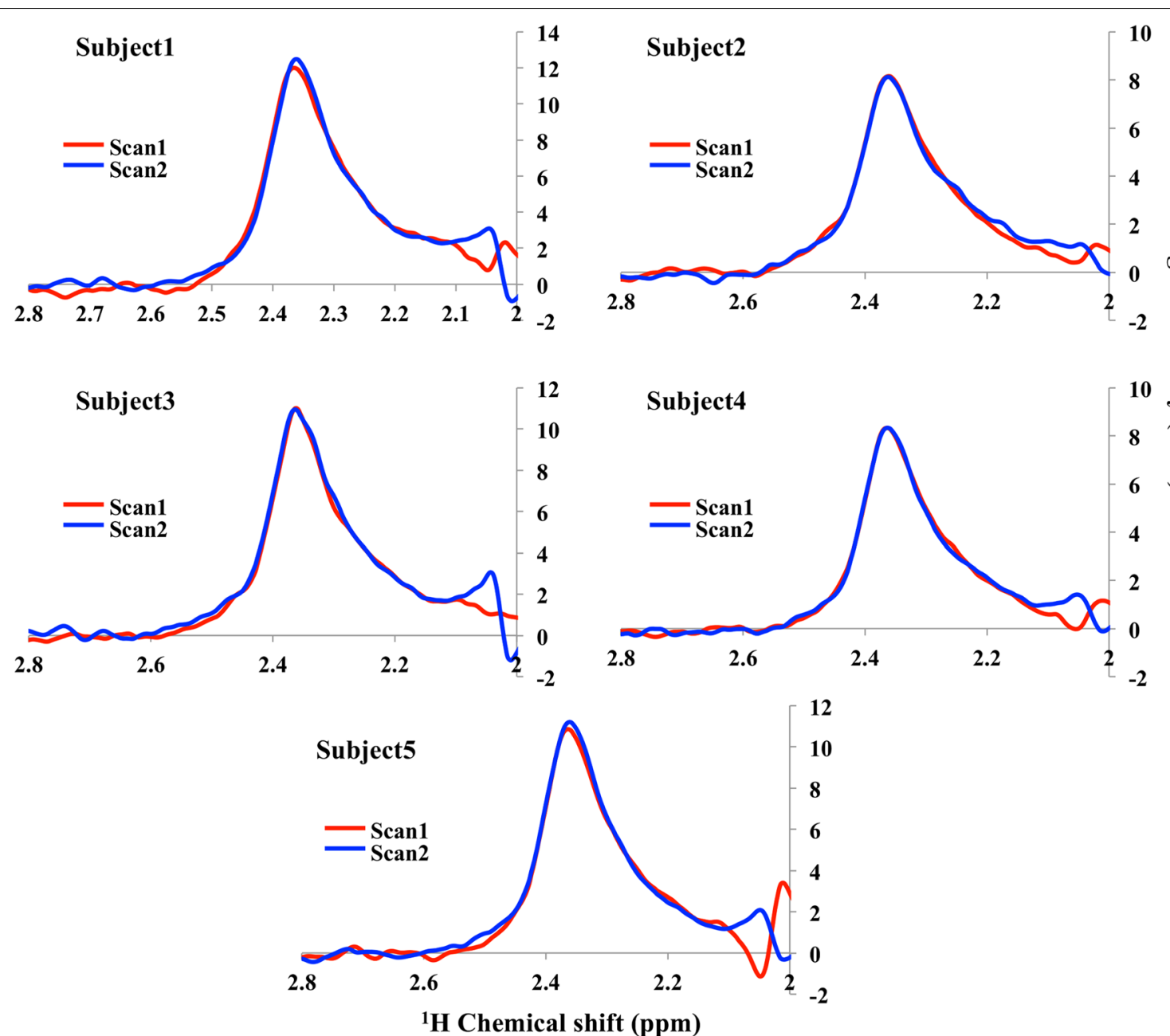

Fig. 7 FAMOUS Glu spectra of scan1 (red) and scan2 (blue) of each volunteer are shown for all the five volunteers participated in the study

Table 1 Absolute variations in Glu concentrations for all the scans from FAMOUS Glu spectra, analyzed with inhouse developed software

\begin{tabular}{lccl}
\hline Subject no. & Glu (mM) & $\begin{array}{l}\text { Abs Glu variation } \\
\text { b/n scans }\end{array}$ \\
\cline { 2 - 3 } & Scan 1 & Scan 2 & \\
\hline 1 & 8.99 & 8.92 & 0.07 \\
2 & 9.35 & 9.2 & 0.15 \\
3 & 8.93 & 9.18 & 0.25 \\
4 & 10.44 & 10.28 & 0.16 \\
5 & 10.08 & 10.32 & 0.24 \\
\hline
\end{tabular}

absolute change in glutamate estimation observed from this voxel is $<0.3 \mathrm{mM}$ (ICC 0.961; $95 \%$ CI LB 0.745, UB 0.996; $\mathrm{p}<0.0005$ ).

\section{Discussion}

We have successfully demonstrated the feasibility of FAMOUS SVGS with studies on glutamate phantoms at 7.0T. The major advantage of this method is the fully automated processing with no user intervention for either phasing or baseline correction. We have also shown that we can estimate Glu concentrations in vivo from the data obtained from healthy subjects by FAMOUS SVGS with an intra-subject variability of less than $0.3 \mathrm{mM}$. This is most likely due to the simplification of the spectrum with negligible baseline and reduction of lipid/macromolecular contamination. We do find that the Glu concentrations in the occipital cortex from the FAMOUS SVGS are comparable to the previously published results from human brain autopsy study [24].

One of our goals in the current study was to bring down the scan-to-scan variation of Glu measurements to 
less than $0.5 \mathrm{mM}$. This objective was achieved with the FAMOUS SVGS method for our voxel of interest.

Although the FAMOUS SVGS method seems to be simplistic with the incorporation of a frequency selective inversion pulse, one might raise the following concern: (i) Effect of $\mathrm{T}_{1}$ recovery of Glu due to delay between inversion pulse and PRESS sequence: The reported $\mathrm{T}_{1}$ values of the metabolites at $7.0 \mathrm{~T}$ were on the order of 1.21-1.83 s [25]. Therefore, the $\mathrm{T}_{1}$-weighting of the MR signals from the $12 \mathrm{~ms}$ delay (half the inversion pulse width $=10 \mathrm{~ms}$ and gradient crusher $=2 \mathrm{~ms}$ ) between the inversion pulse and the PRESS sequence is negligible $(<1 \%)$ (ii) Macromolecule/lipid contamination at $2.35 \mathrm{ppm}$ : This has been accounted in our method.

For the voxels of interest where local $\mathrm{B}_{0}$ inhomogeneity is large, we expect the accuracy of quantification to deteriorate due to poor shimming. Additionally, this method is highly sensitive to subject motion and hence we needed to immobilize the head with proper padding.

An alternate strategy we have considered was the use of long echo time without any inversion to reduce the macromolecule/lipid content from the control in vivo spectra. But the complicated coupling pattern of the glutamate peak poses additional quantification issues due to which we preferred to go along with the short echo spectroscopy with FAMOUS SVGS.

Although we have demonstrated the performance of FAMOUS SVGS in combination with in-house developed automated post-processing method at 7.0T, it can be easily implemented on animal imaging scanners $\geq 7.0 \mathrm{~T}$.

\section{Conclusions}

In conclusion, FAMOUS SVGS can be one of the useful ${ }^{1} \mathrm{H}$ MRS tools in any studies that measure regional glutamate changes in brain. The significant improvement in repeatability of the measurement has implications in quantifying relatively smaller change in Glu, reducing the number of subjects and time points needed in a clinical trial and thereby contributing to significant savings in study cost and time.

\footnotetext{
Abbreviations

${ }^{1} \mathrm{H}$ MRS: proton magnetic resonance spectroscopy; Glu: glutamate; SVGS: single voxel glutamate spectroscopy; SNR: signal-to-noise ratio; GABA: gamma-amino butyric acid; OVS: outer volume suppression; FSI: frequency selective inversion; PRESS: point resolved spectroscopy; RF: radio frequency; OCC: occipital cortex.

\section{Authors' contributions}

RPRN contributed to conception and design of the study, performed the experiments and wrote the manuscript. $\mathrm{HH}$ contributed to coding the pulse sequence, manuscript writing and editing. RR provided conception and contributed to manuscript editing. All authors read and approved the final manuscript.
}

Acknowledgements

The author thanks Dr. Tiejun Zhao from Siemens for providing FASTMAP WIP.

Competing interests

The authors declare that they have no competing interests.

\section{Funding}

This project was supported by the National Institute of Biomedical Imaging and Bioengineering of the National Institute of Health through Grant Number P41-EB015893 and the National Institute of Neurological Disorders and Stroke through Grant Number R01NS087516.

Received: 5 May 2016 Accepted: 11 July 2016

Published online: 26 July 2016

\section{References}

1. Sutoo D, Akiyama K, Yabe K. Quantitative maps of GABAergic and glutamatergic neuronal systems in the human brain. Hum Brain Mapp. 2000;11:93-103.

2. Auer DP, Pütz B, Kraft E, Lipinski B, Schill J, Holsboer. Reduced glutamate in the anterior cingulate cortex in depression: an in vivo proton magnetic resonance spectroscopy study. Biol Psychiatry. 2000;47:305-13.

3. Benedetti F, Calabrese G, Bernasconi A, Cadioli M, Colombo C, Dallaspezia S, Falini A, Radaelli D, Scotti G, Smeraldi E. Spectroscopic correlates of antidepressant response to sleep deprivation and light therapy: a 3.0 Tesla study of bipolar depression. Psychiatry Res. 2009;173:238-42.

4. Griffith HR, den Hollander JA, Okonkwo OC, O'Brien T, Watts RL, Marson DC. Brain metabolism differs in Alzheimer's disease and Parkinson's disease dementia. Alzheimers Dement. 2008:4:421-7.

5. Gutzeit A, Froehlich JM, Hergan $\mathrm{K}$, et al. Insula-specific ${ }^{1} \mathrm{H}$ magnetic resonance spectroscopy reactions in heavy smokers under acute nicotine withdrawal and after oral nicotine substitution. Eur Addict Res. 2012;19:184-93.

6. Hasler G, van der Veen JW, Tumonis T, Meyers N, Shen J, Drevets WC. Reduced prefrontal glutamate/glutamine and gamma-aminobutyric acid levels in major depression determined using proton magnetic resonance spectroscopy. Arch Gen Psychiatry. 2007;64:193-200.

7. Kakeda S, Korogi Y, Moriya J, Ohnari N, Sato T, Ueno S, Yanagihara N, Harada M, Matsuda T. Influence of work shift on glutamic acid and gamma-aminobutyric acid (GABA): evaluation with proton magnetic resonance spectroscopy at 3T. Psychiatry Res. 2011;192:55-9.

8. Kegeles LS, Mao X, Stanford AD, et al. Elevated prefrontal cortex $\gamma$-aminobutyric acid and glutamate-glutamine levels in schizophrenia measured in vivo with proton magnetic resonance spectroscopy. Arch Gen Psych. 2012;69:449-59.

9. Marsman A, van den Heuvel MP, Klomp DW, Kahn RS, Luijten PR, Pol HHE. Glutamate in schizophrenia: a focused review and meta-analysis of ${ }^{1} \mathrm{H}$ MRS studies. Schizophr Bull. 2013;39:120-9.

10. Petroff OA, Rothman DL, Behar KL, Mattson RH. Initial observations on effect of vigabatrin on in vivo ${ }^{1} \mathrm{H}$ spectroscopic measurements of gamma-aminobutyric acid, glutamate, and glutamine in human brain. Epilepsia. 1995;36:457-64.

11. Price RB, Shungu DC, Mao X, Nestadt P, Kelly C, Collins KA, Murrough JW, Charney DS, Mathew SJ. Amino acid neurotransmitters assessed by proton magnetic resonance spectroscopy: relationship to treatment resistance in major depressive disorder. Biol Psychiatry. 2009;65:792-800.

12. Sanacora G, Gueorguieva R, Epperson CN, Wu YT, Appel M, Rothman DL, Krystal JH, Mason GF. Subtype-specific alterations of gamma-aminobutyric acid and glutamate in patients with major depression. Arch Gen Psychiatry. 2004;61:705-13.

13. Schmaal L, Veltman DJ, Nederveen A, van den Brink W, Goudriaan AE. $\mathrm{N}$-acetylcysteine normalizes glutamate levels in cocaine-dependent patients: a randomized crossover magnetic resonance spectroscopy study. Neuropsychopharmacology. 2012;37:2143-52.

14. Yucel M, Lubman DI, Harrison BJ, et al. A combined spectroscopic and functional MRI investigation of the dorsal anterior cingulated region in opiate addiction. Mol Psychiatry. 2007;12:691-702. 
15. Stephenson MC, Gunner F, Napolitano A, Greenhaff PL, Macdonald IA, Saeed N, Vennart W, Francis ST, Morris PG. Applications of multi-nuclear magnetic resonance spectroscopy at 7T. World J Radiol. 2011;3:105-13.

16. Cai K, Nanga RPR, Lamprou L, Schinstine C, Elliott M, Hariharan H, Reddy $\mathrm{R}$, Epperson CN. The impact of gabapentin administration on brain GABA and glutamate concentrations: a $7 \mathrm{~T}^{1} \mathrm{H}-\mathrm{MRS}$ study. Neuropsychopharmacology. 2012;37:2764-71.

17. Terpstra M, Cheong I, Lyu T, Deelchand DK, Emor UE, Bednarik P, Eberly $\mathrm{LE}, \mathrm{Oz} \mathrm{G}$. Test-retest reproducibility of neurochemical profiles with shortecho, single-voxel MR spectroscopy at 3T and 7T. Magn Reson Med. 2015. doi:10.1002/mrm.26022

18. Lally N, An L, Banerjee D, Niciu MJ, Luckenbaugh DA, Richards EM, Roiser JP, Shen J, Zarate CA, Nugent AC. Reliability of 7T (1)H-MRS measured human prefrontal cortex glutamate, glutamine, and glutathione signals using an adapted echo time optimized PRESS sequence: a between- and within-sessions investigation. J Magn Reson Imaging. 2016;43:88-98.

19. Pauly J, LeRoux P, Nishimura D, Macovski A. Parameter relations for the Shinnar-Le Roux selective excitation pulse design algorithm [NMR imaging]. IEEE Trans Med Imaging. 1991;10:53-65.
20. Gruetter R. Automatic, localized in vivo adjustment of all first-and second-order shim coils. Magn Reson Med. 1993;29:804-11.

21. Gruetter R, Tkac I. Field mapping without reference scan using asymmetric echo-planar techniques. Magn Reson Med. 2000;43:319-24.

22. Wolf DH, Satterhwaite TD, Loughead J, Pinkham A, Overton E, Elliott MA, Dent GW, Smith MA, Gur RC, Gur RE. Amygdala abnormalities in first-degree relatives of individuals with schizophrenia unmasked by benzodiazepine challenge. Psychopharmacology. 2011;218:503-12.

23. Landis JR, Koch GG. The measurement of observer agreement for categorical data. Biometrics. 1977;33:159-74.

24. Perry TL, Berry K, Hansen S, Diamond S, Mok C. Regional distribution of amino acids in human brain obtained at autopsy. J Neurochem. 1971;18:513-9.

25. Xin L, Schaller B, Mlynarik V, Lu H, Gruetter R. Proton T1 relaxation times of metabolites in human occipital white and gray matter at 7T. Magn Reson Med. 2013;69:931-6.

\section{Submit your next manuscript to BioMed Central and we will help you at every step:}

- We accept pre-submission inquiries

- Our selector tool helps you to find the most relevant journal

- We provide round the clock customer support

- Convenient online submission

- Thorough peer review

- Inclusion in PubMed and all major indexing services

- Maximum visibility for your research

Submit your manuscript at www.biomedcentral com/submit 